\title{
La huella ecológica, indicador de sostenibilidad ambiental y social
}

\author{
José Claudio Guarín Calle \\ claudioguarin@hotmail.com \\ Institución Educativa Las Guacas Corinto Cauca. \\ Maestrante Pedagogìa Ambiental para el Desarrollo Sostenible,

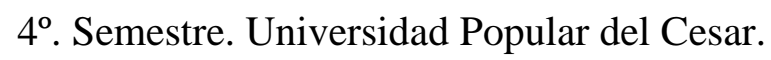 \\ Celular y whatsapp: 3104942068 \\ Yoorsoly Vitoncó Orozco \\ yoor10@hotmail.com \\ Institución Educativa Técnico Tunia Piendamò Cauca. \\ Maestrante Pedagogìa Ambiental para el Desarrollo Sostenible, \\ $4^{\circ}$. Semestre. Universidad Popular del Cesar. \\ Celular y whatsapp: 3163563293
}

\section{RESUMEN}

Ultimamente el planeta tierra ha experimentado diversos cambios y manifestaciones que ponen en jaque la vida de hombre y de todas las especie en general; una realidad que se asocia principalmente al abuso en la utilización de los recursos para satisfacer la industria o los mercados de bienes y servicios. El presente articulo tuvo como objetivo, fomentar la responsabilidad social en la huella ecológica, a partir del análisis de un caso particular en la Comunidad Educativa Técnico Tunía de Piendamó. La metodología utilizada tuvo un enfoque cualitativo, donde se tomó como referente el tipo investigación aplicada IAP, la cual comprendió la aplicación de una entrevista en profundidad al caso donde participó un docente de la institución. Igualmente se utilizó la herramienta Global Footprint Network, para evaluar los indicadores mas importantes, acorde al objetivo propuesto. Se concluye que la huella ecológicas del caso de estudio se ubica en el 4.3 muy por encima del valor nacional de 1.9 y de la internacional que se ubica en 2.87. La reducción de la huella ecológica, implica un proceso de transformación de las actividades y la formación de hábitos para el buen manejo de los residuos y desechos.

Palabras claves: huella ecològica; desarrollo sostenible; indicador de sostenibilidad; medio ambiente 
Guarín Calle y Vitoncó Orozco...

\title{
Ecological footprint, environmental and social impact indicator
}

\begin{abstract}
Lately the land plant has undergone various changes and manifestations that put in check the life of man and of all species in general; a reality that is mainly associated with the use of resources to satisfy the industry or the markets for goods and services. The objective of this article was to promote social responsibility in the ecological footprint, based on the analysis of a particular case in the Tunía de Piendamó Technical Educational Community. The methodology used had a qualitative approach, where the IAP applied research type was taken as a reference, which included the application of an in-depth interview to the case where a teacher from the institution participated. Likewise, the Global Footprint Network tool was used to evaluate the most important indicators, according to the proposed objective. It is concluded that the ecological footprint of the case study is located at 4.3 , well above the national value of 1.9 and the international one, which is located at 2.87. The reduction of the ecological footprint implies a process of transformation of activities and the formation of habits for the proper management of waste and waste.
\end{abstract}

Keywords: ecological footprint; sustainable development; sustainability indicator; environment

Artículo recibido: 15 enero 2022 Aceptado para publicación: 08 febrero 2022 Correspondencia: claudioguarin@ hotmail.com Conflictos de Interés: Ninguna que declarar 


\section{INTRODUCCIÓN}

Históricamente la sociedad ha vivido una situación crítica a nivel ambiental, toda vez que los conceptos y teorías del modelo capitalista dominante, están pensados desde una cultura superior quien promueve una forma única de ver el mundo, un proyecto excluyente que deja por fuera importantes actores sociales como las mujeres, los niños, los ancianos, pero sobre todo, ha desconocido las riquezas naturales y culturales de los pueblos minoritarios (Torbisco, 2000). Al respecto Bauman (2000) expresa que el hombre vive en una "sociedad líquida", marcada por el consumo desmedido, influenciado por los medios de comunicación, donde el deseo de estar a la moda y el interés económico han dado lugar a un fenómeno que invita a consumir y consumir, lo que ha promovido el desarraigo de los recursos naturales incluso por encima de la capacidades del planeta.

En este sentido, las conductas consumista han generado un escenario perfecto para la explotación indiscriminada de los resursos naturales y por ende el surgimiento de nuevos desarrollos tecnológicos que poco le aportan al medio ambiente, sino que van en detrimento de este último causando serias afectaciones en los ecosistemas, (Salazar, 2018). De hecho, la transformación del suelo, la fabricación de bienes a gran escala, esta llevando al agotamiento de recursos no renovables como el agua, las flora, minerales entre otros, que son vitales para la supervivencia de las especies, ha llevado a la dregradación de las capas del subsuelo y los ecosistemas en general, afectando directamente las especies nativas de la fauna y flora.

Por consiguiente, los humanos en las últimas décadas hemos sido testigos de las diferentes manifestaciones ambientales como desbordamiento de ríos, las lluvias intensas, temperaturas sofocantes, movimientos sísmicos, entre otros fenómenos naturales que viene afectando considerablemente la vida en el planeta, impactando especialmente en los grupos menos favorecidos como campesinos, agricultores, indígenas o aquellos asentamientos que se ubican en los centros periféricos de las grandes ciudades. Esta realidad ha visto agravada por la falta de hábitos frente al manejo de los residuos y desechos, lo que ha dado lugar a la proliferación de vectores y roedores que constituyen un riesgo para la salud de los habitantes, sobre todo por la liberación de gases y partículas de efecto invernadero, (Jaramillo y Zapata, 2008).

Así mismo, se vislumbra un total desaprovechamiento de los materiales que pueden ser reincorporados a las cadenas productivas como el plástico, papel y el vidrio, pero quizá 
lo que más llama la atención es el poco interés de aprovechar los residuos orgánicos, desconociendo su potencial en la producción agrícola, siendo esta última el principal renglón económico de los habitantes. Lo anterior, pone en evidencia la falta de estrategias sobre el manejo de los residuos por parte de los individuos y el Estado, lo cual se asocia a varios aspectos, pero en especial a la falta de un factor motivacional que despierte el interés por cuidar los ecosistemas y el medio ambiente en general.

Frente a esta realidad, la corriente estadística de la huella ecológica, plantea un tipo de gestión ambiental desde una perspectiva transformadora, crítica, reflexiva y contextualizada, donde todas las acciones ambientales se desarrollen acorde a las riquezas culturales de las comunidades, a fin de que se alcance una perspectiva de cambios que permita promover hábitos ambientalmente saludables desde y para el contexto. De hecho la huella ecológica es entendida como un concepo general e individual, que muestra sus repercusiones de las actividades del hombre, en los ecosistemas, en la medida que arroja información importante para dar soluciones a la crisis socio-ambiental, desde una posición política, pues, es un indicador que estima el impacto real de la actividad humana sobre el entorno natural y la misma sociedad, (Martínez, 2007).

Asi mismo, se trata de un indicador del impacto que ejerce una cierta comunidad humana sobre su entorno ecológico en el que se relacionan los elementos naturales tales como sabanas, bosques, ecosistemas acuáticos, con los hábitos de consumo y el nivel de vida de un grupo humano, no solo el área de territorio ecológicamente necesaria para producir los recursos utilizados, sino también el área necesaria para asimilar los residuos, las basuras y la contaminación producida por una población determinada con un nivel de vida específico durante un tiempo indefinido, (Bohórquez, 2006). Desde esta perspectiva, se busca impulsar una política pública incluyente, que respondan a una verdadera transformación social para poder afrontar las verdaderas necesidades de la población en el marco de un modelo de desarollo sostenible.

En otras palabras, la huella ecológica constituye una metodología para visualizar de manera cuantitativa el consumo de recursos ambientales, lo cual resulta fundamental para el desarrollo de la gestión ambiental, ademàs porque aporta instrumentos que estimulen y viabilicen esta tarea, dado que mide la cantidad de superficie de tierra biológicamente productiva necesaria para producir bienes y servicios que consumimos, y absorber los 
residuos que producimos, además de que permite medir la presión ejercida por los humanos sobre la naturaleza, (Pérez et al., 2015).

Para entender el concepto, se puede partir de algunas observaciones: La Tierra tiene un área total de 51 mil millones de hectáreas, sólo 11.4 mil millones de ellos son biológicamente productivos: los campos cultivables, los bosques que absorben $\mathrm{CO} 2$, los océanos que producen los peces, etc. pero no los desiertos, por ejemplo, donde no es cultivable (Gàndara, 2011). En esta area se distribuyen los 7.700 millones de personas, una población que va en aumento día tras día y va dejando una huella ecológica sin precendentes, en la medida que sus actividades realizadas a nivel individual, familiar o empresarial implican la utilización de enormes cantidades de recursos, muchos de ellos no renovables, además de la emisión de materiales contaminantes, (McAuliffe y Khadria, 2019).

Ahondando en el concepto, la huella ecológica representa una estimación de la superficie necesaria para una persona o grupo de personas para producir lo que consumen y lo que rechazan, se expresa en unidad de superficie: hectárea $\left(10.000 \mathrm{~m}^{2}\right)$. Por ello, si hubiera una distribución equitativa de área biológicamente productiva de la tierra entre todos sus habitantes, cada uno tendría un "derecho" a 1,75 has. Si se reserva un espacio para la diversidad biológica a la "naturaleza salvaje", queda alrededor de 1,6 hectáreas para satisfacer sus necesidades a largo plazo. Sin embargo, en promedio, cada individuo "consume" ya 2,2 hectáreas, lo cual resulta crítico si se tiene en cuenta que la demanda de bienes y servicios por parte de los habitantes supera de mucho la oferta del planeta, (Sánchez et al., 2019).

A esta realidad se suma la distrubución inequitativa de los resursos naturales, por ejemplo: en promedio, un europeo necesitaría 4,5 hectáreas, mientras que un norteamericano necesitaría 6,6 hectáreas y un africano 2,7 (Garrett, 2021). Asi mismo, es de aclarar que la huella ecológica de los países desarrollados y de la población mundial sigue creciendo: el área productiva disponible por persona se reduce así día tras día y por ende causa seria afectaciones al sistema que sostiene la vida de la Tierra, de ahí la inmensa responsabilidad que tiene el hombre sobre sus hombros.

Aunque Colombia presenta una huella ecológica relativamente baja, (1,9 has/persona), no es posible afirmar que dicha cifra indique la existencia de una sociedad ambientalmente sostenible, pues según los datos de la Global Footprint Network, en 2016 
(medición de 2012), el país se ubicaba en el puesto 92 entre 150 países analizados por su huella ecológica, mientras que por su biocapacidad ocupaba el puesto14, (Palomares, 2018). La mayor proporción de este valor estuvo representada por las emisiones de carbono que tuvieron un auge en los años ochenta-noventa, alcanzó los 0,5 GHA y que tienden a tener un aumento para el año 2012 de hasta 0,7 GHA, (Palomares, 2018).

Expuesto lo anterior, el presente articulo tiene como objetivo general determinar cómo la huella ecológica, permite fomentar la responsabilidad social, impulsar un adecuado aprovechamiento y disposición de los recursos existentes, ademas de analizar un caso particular sobre el cálculo de la medición de dicho indicador, con el fin de para promover su comprensión en la Comunidad Educativa Técnico Tunía de Piendamó en el marco de un proceso de desarrollo sostenible que pueda ser replicado a nivel institucional y comunitario.

El presente proyecto es de gran importancia porque permite generar conciencia ambiental en todos los miembros de la comunidad educativa mediante la capacitación y formación ambiental sobre el aprovechamiento y disposición de los residuos sólidos inorgánicos, generados por la comunidad educativa Técnico Tunía, que beneficie los entornos escolares y disminuya la contaminación ambiental. El proyecto se considera innovador porque involucra al estudiantes y su familia en el proceso de reciclaje y comprometer a todos los docentes, administrativos y líderes comunitarios implica hacer trabajo en equipo, para viabilizar nuevas posibilidades y propuestas que permitan mantener un entorno limpio, agradable y saludable para la comunidad educativa.

En consecuencia, este proyecto tiene un impacto social positivo porque beneficia a todas las comunidades educativas, la población y el entorno aledaño a la institución, porque motivada la formacion hábitos ambientales saludables, a partir de el manejo adecuado de los residuos sólidos en caminados la mitigación de la contaminación ambiental, pero al mismo tiempo a la reutilización de los materiales recuperables como madera, plástico, metales, vidrios, entre otros. En otras palabras el proyecto está encaminado a que, desde la familia del estudiante recicle y sobre todo tome conciencia de no arrojar la basura, sino, que se cree una cultura para el mejoramiento del ambiente escolar, familiar y comunitario. De igual manera, el presente estudio tiene un gran valor teórico, dado que, el cual está inscrito dentro del Proyecto Ambiental Escolar (PRAE), a traves del cual se promueve el análisis y la comprensión del problema, donde se evalùa la influencia de la propuesta 
metodológica al capacitar en escuela de padres y al crear vigías ambientales en los cambios de actitud frente a la buena disposición de los residuos sólidos inorgánicos (Plástico). Asi mismo, permite orientar a la comunidad educativa para mejorar las prácticas educativas frente a la protección del medio ambiente y el ecosistema en general, acorde con la realidad que viven los estudiantes y la comunidad.

\section{MATERIALES Y MÉTODOS}

Para llevar a cabo la presente investigación, fue preciso recurrir al enfoque cualitativo, desde la perspectiva de Hernández, Fernández y Baptista (2006), la cual tiene como finalidad especial la obtención de los hallazgos, el análisis y la interpretación de los mismos, para llegar a su comprensión y difusión, en último término, la mejora de la realidad investigada. Este enfoque fue de gran utilidad dado que, permitió obtener información con base en las cualidades de los participantes lo que condujo a resultados concretos, para determinar cómo la huella ecológica y el aprovechamiento de los recursos naturales de manera óptima, permite fomentar el desarrollo sostenible individual y colectivamente.

El paradigma de investigación es Socio-Crítico, el cual considera que el conocimiento se construye siempre por intereses que parten de las necesidades de las comunidades; pretende la autonomía racional y liberadora del ser humano; y se consigue mediante la capacitación de los sujetos para la participación y transformación social, (Martínez, 2004). Así mismo este enfoque utiliza la autorreflexión y el conocimiento interno y personalizado para que cada quien tome conciencia del rol que le corresponde dentro del grupo; para ello se propone la crítica ideológica y la aplicación de procedimientos del psicoanálisis que posibilitan la comprensión de la situación de cada individuo, descubriendo sus intereses a través de la crítica, (Martínez, 2004).

En lo que refiere al tipo de estudio, se recurre a Investigación, Acción, Participación, (IAP); pues como su nombre lo indica, el investigador participa de manera activa en la realidad que se investiga, para identificar sus propias necesidades (diagnóstico) y por ende lograr la transformación del contexto de los participantes (Balcazar, 2003). Este tipo de investigación aporta un carácter protagónico a las comunidades en la transformación social y ambiental que necesitan, y el problema a investigar es delimitado, atendido, analizado y confrontado por la propia comunidad afectada, puesto que el papel del 
investigador es el de ser dinamizador y orientador del proceso, produciéndose una relación con la comunidad.

La población contempla los Integrantes de la comunidad de la Institución Educativa Técnico Tunía del Municipio de Piendamó-Cauca. (Estudiantes, docentes, administrativos, padres de Familias y líderes comunitarios). Los estudiantes de la Institución se distribuyen en cuatro sedes educativas para un total de setecientos siete (707) estudiantes. Los docentes y directivos docentes suman un total de cuarenta y ocho (48), son diez (10) líderes Comunitarios, los cuales se escogen por el método de conveniencia.

Para la elección de la muestra se utiliza el muestreo no probabilístico estratégico, también conocido como muestreo por conveniencia, debido a que la selección de las unidades muestrales responden a los criterios del investigador (Hernández et al., 2006). En este sentido, el número de participantes, se define teniéndose en cuenta la naturaleza del fenómeno a investigar (Inadecuado aprovechamiento y disposición de los residuos sólidos inorgánicos y la poca responsabilidad en la Huella Ecológica en la comunidad Educativa Técnico Tunía de Piendamó), Es decir un total, de ciento treinta y dos (132), con este número se estima que se puede recoger la información necesaria para sacar adelante la investigación.

En lo que se refiere a las variables, esta fueron establecidas a partir de los objetivos propuestos, entre los que se destacan, responsabilidad ambiental, huella ecológica, aprovechamiento y disposición de los residuos sólidos inorgánicos. Estas categorías constituyen la columna vertebral para la elaboración de los instrumentos de recolección de los datos en especial la entrevisa semiestructurada aplicada a estudiantes, a docentes, a padres de familia y a lideres de la comunidad. Asi mismo estas categorías facilitaron la estructuración de la información obtenida en las obsevaciones participantes realizadas al caso de estudio, las cuales fueron registradas en los diarios de campo.

Para el análisis del caso particular del càlculo de la huella ecológica se tomó como referencia las publicaciones más recientes del Global Footprint Network en torno al cálculo de la huella ecológica de las naciones (WWF, 2019). Como herramienta de cálculo se empleó la calculadora de huella ecológica personal disponible en la página web Estrategia UPB Sostenible (2019) de la Universidad Pontificia Bolivariana de Colombia, denominada footoprint calculator, en la cual se pueden ingresar la cantidad de bienes 
consumibles màs relevantes. como el uso de la tierra, la energía el consumo de alimentos organicos, la utilización de combustibles y la generación de basuras.

Para el análisis de la información obtenida se recurre a la técnica de triangulación de codificación de la información. Esta fase consistió en asignar un código a los diferentes relatos de tal manera que puedan ser agrupados acorde a su relación o similitud. Adicionalmente, se realizó la Categorización de la información para reducir los datos, es decir, escribirlos de manera conceptual, para que puedan ser traducidos en categorías para realizar comparaciones y contrastes.

En este sentido, se utilizó la técnica de análisis denominada triangulación de resultados, la cual consiste en tomar los relatos más representativos de cada categoría, y contrastarlos con la teoría consultada y la opinión del investigador, dando como resultado nuevos hallazgos que construirán la columna vertebral de las conclusiones del estudio.

\section{RESULTADOS}

El presente apartado expone los resultados obtenidos en el análisis de un caso de aplicación de la huella ecológica en la institución, a fin de brindar una mayor comprensión de el concepto, pero sobre todo de las categorías mas importantes como: el consumo de alimentos, la utilización de la energía eléctrica, la economía del combustible, la generación de basuras y el porcentaje de electricidad, como se expone a continuación:

\section{Al consumo de alimentos no procesados, no envasados o cultivados localmente}

En esta categoría se observa un valor que se ubica en el $20 \%$, el cual resulta altamente representativo, como se muestra en la figura1.

Figura 1. Porción de alimentos orgánicos que consume

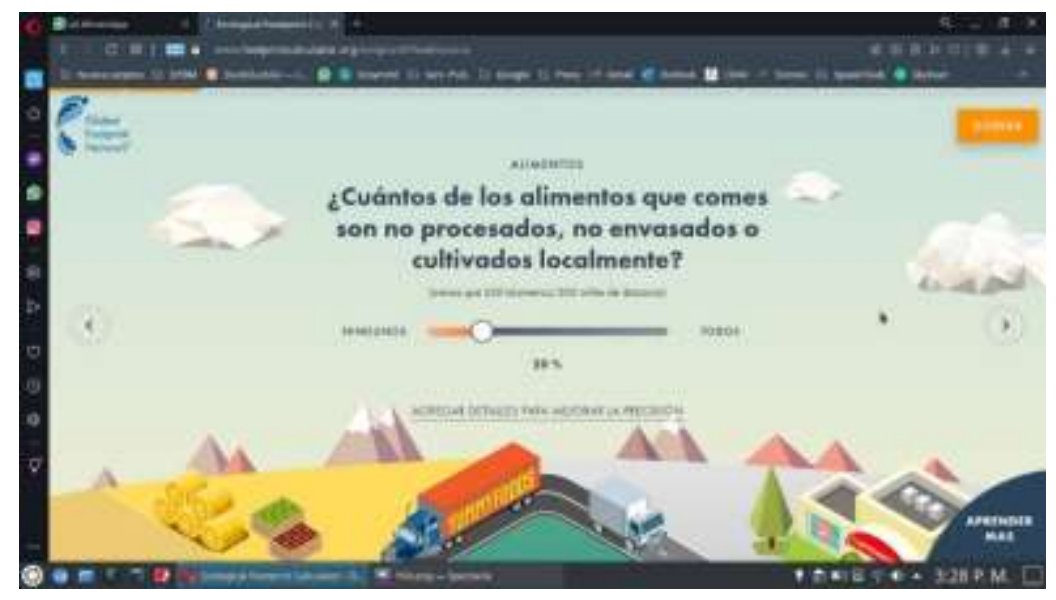

Fuente: propia a partir de la herramienta Global Footprint Network (2019) 
Lo anterior, resulta relevante dado que un alto consumo de productos procesados o envasados han pasado por un proceso industrial donde muy probablemente se utilizaron grandes cantidades de energía, materias primas, entre otros recursos y por ende un alto costo en materia ambiental. Es aquí donde se requiere formar hábitos para una alimentación saludable, es decir aquellos alimentos producidos mediante técnicas orgánicas y artesanales, a fin de evitar deterioros a los recursos del planeta.

\section{Consumo de electricidad}

En este sentido, se observa que el consumo para este caso particular no es muy representativo, sin embargo èste se encuentra por encima del promedio, como se muestra en la figura 2.

Figura 2. Consumo de energía en el hogar



Fuente: propia a partir de la herramienta Global Footprint Network (2019)

Según los resultados anteriores, se observa que el consumo de electricidad también es importante analizarlo porque este recurso se produce mediante la utilización de otros recursos no renovabes como el gas natural, carbón entre otros que representan un alto costo ambiental.

\section{La generación de basuras}

La generación de basura es un aspecto importante porque esta contribuye significativamente en los procesos de contaminación ambiental, especialmente cuando no se cuenta con un mecanismo eficiente de manejo. En este sentido, se observa que este indicador se encuentra en una medida muy equilibrada con la que generan los vecinos de la comunidad como se muestra en la figura 3. 
Figura 3. Basura generada en comparación con los vecinos

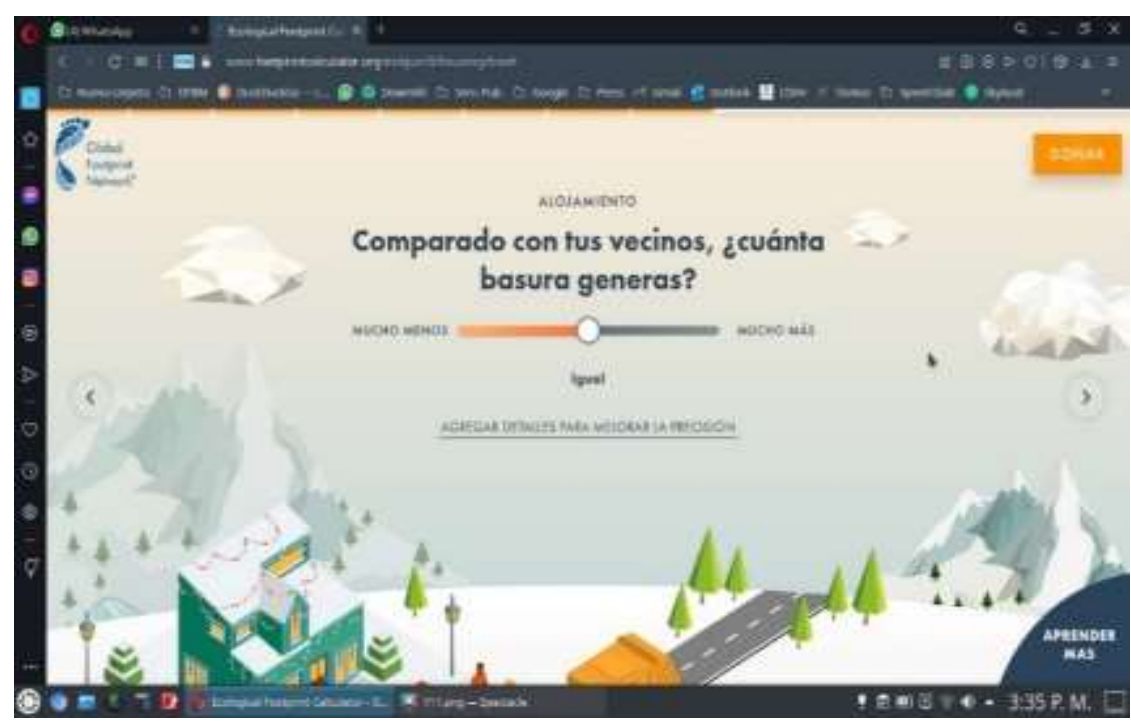

Fuente: propia a partir de la herramienta Global Footprint Network (2019)

Ante este resultado es evidente la necesidad de estrategias de gestión de los residuos para evitar que estos vayan a los centros desecho, y se contribuya en la mitigación de la contaminación del entorno, en la medida que se evita la proliferación de vectores que además pueden causar daños en la salud.

\section{Consumo de combustible}

En esta categoría se observa que no hay una economía eficiente en el consumo de combustible lo que esta propiciando la generación de CO2 y por ende aumentando la huella ecológica como se observa en la figura 4.

Figura 4. Economía en el combustible del vehículo.

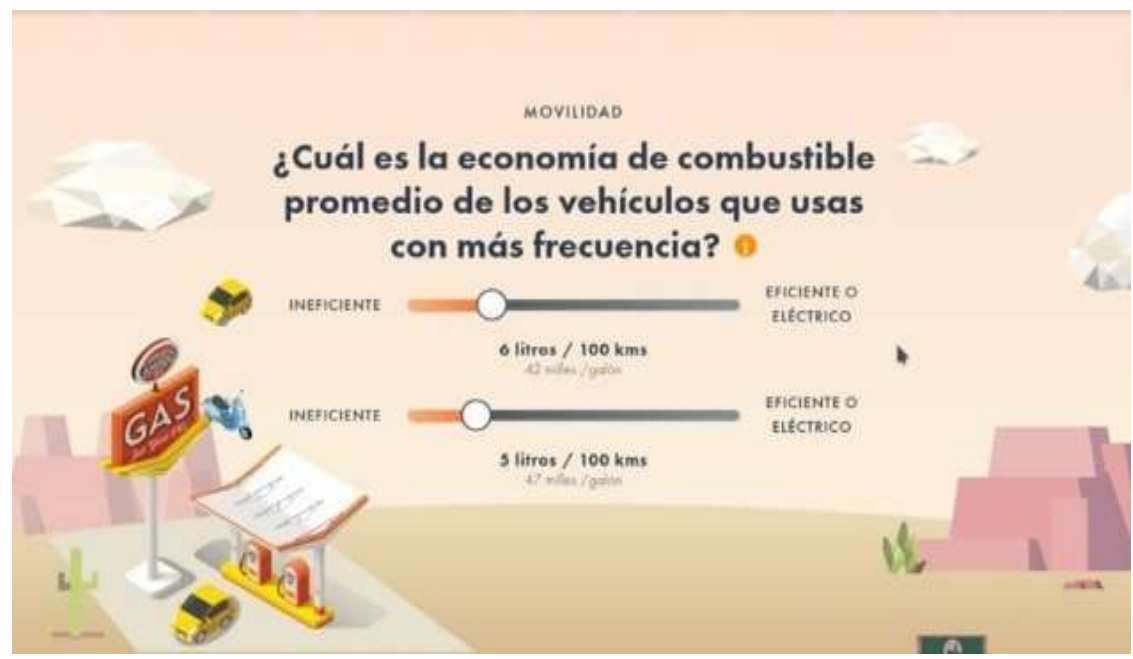

Fuente: propia a partir de la herramienta Global Footprint Network (2019) 
El ahorro de combustibles fósiles es un asunto necesario en la actualidad, dado que se reducen las emisiones de $\mathrm{Co} 2$ y por ende se contribuye preservación de la calidad del aire, y sobre todo a la mitigación de la capa de ozono.

\section{Número de personas que viven en el hogar}

En esta categoría se encontró que no hay hacinamiento, dado que solo habitan tres personas, aunque el hogar tambien presenta un espacio pequeño como se observa en la figura 5 .

\section{Figura 5. Personas que habitan en el hogar}

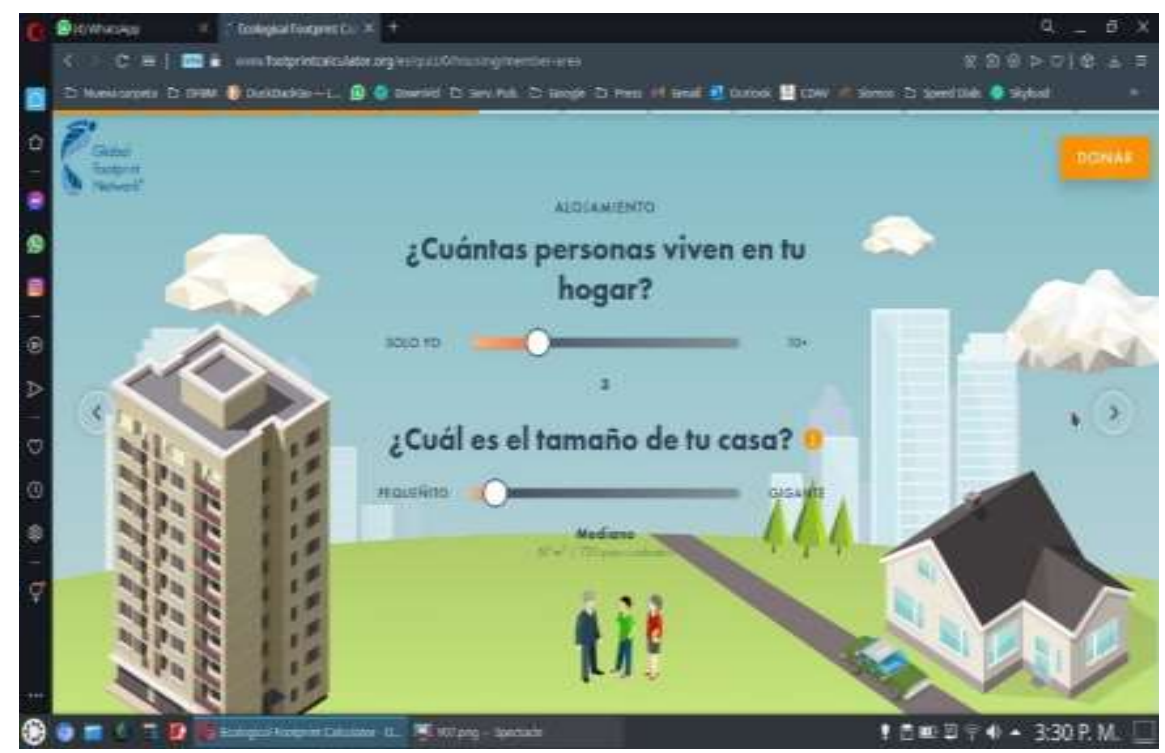

Fuente: propia a partir de la herramienta Global Footprint Network (2019)

Según estos resultados, es importante mantener una buena relación entre el tamaño de la vivienda, los habitantes de la misma, pues de lo contrario se estarían generando un costo elevado del consumo de servicios.

\section{Resultado general de la huella ecológica}

Una vez ingresada la información de los consumos de bienes y servicios en las diferentes secciones se obtiene el resultado final, el cual para este caso de estudio único de huella ecológica personal fue 4.3 hag, como se aprecia en la figura 6. 
Figura 6. Resultado de la huella ecológica personal para el caso de estudio.

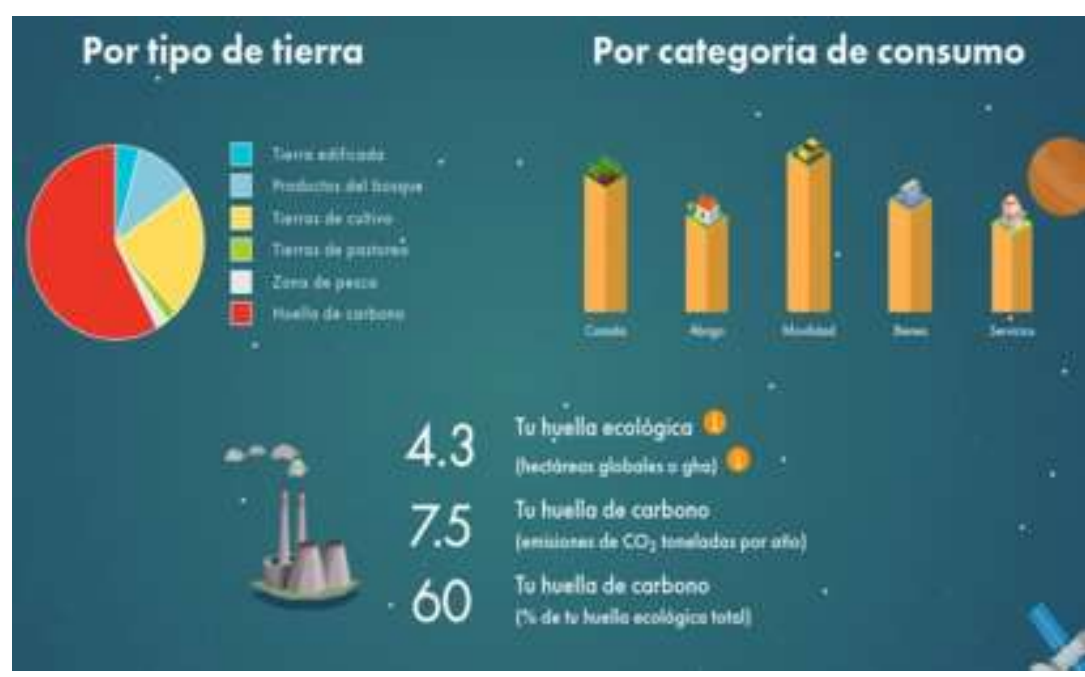

Fuente: propia a partir de la herramienta Global Footprint Network (2019)

Como se observa, el nivel de consumo de este caso único, la huella ecológica de $4.3 \%$, se puede considerar superior a la del mundo (2.87 hag) y superior a la huella de Colombia (1.9 hag), resultado que impactó de gran manera y motiva a reflexionar sobre el estilo de vida y de consumo actual, y a plantearse algunas acciones que pueden disminuir la misma. Si se tiene en cuenta la cantidad de hectáreas globales de la tierra, a cada persona en el mundo le corresponde una huella ecológica de 1.8 hag, por lo que la persona tomada como referencia para este estudio de caso, necesitaría aproximadamente 2.7 tierras.

Por ello se considera que este resultado de la huella ecológica está fuera de los límites de sustentabilidad global, el cual coincide con los resultados, publicado en el sitio Web Estrategia UPB Sostenible (2019) de la Universidad Pontificia Bolivariana de Colombia, donde a nivel de los hogares los principales aportes están dados por el consumo de alimento, consumo de combustibles, por vehículos, transporte y producción de residuos sòlidos. De alli la importancia de promover hábitos de consumo responsable con el fin de lograr reducir este indicador, sobre todo en aquellos ítems que aportan significativamente a la misma, sin afectar significativamente la calidad de vida.

\section{DISCUSIÓN}

La huella ecológica como un indicador estadístico, presenta un gran potencial comunicativo, en la medida que se adapta a diferentes escalas: personal, regional, nacional e internacional, cuyos resultados se expresa en hectárea global (hag) como la unidad de superficie requerida para todos los procesos productivos, los consumos de 
materiales y energía y la absorción de los residuos generados. Estos resultados coinciden con los expuesto por el director general del Fondo Mundial para la Naturaleza (WWF, por sus siglas en inglés) en el Informe Planeta Vivo del (2004), quien manifiesta que "los seres humanos estamos consumiendo los recursos más rápido de lo que la Tierra los puede reponer" (World Wildlife Fund [WWF], 2014, p.14).

En consecuencia el estudio de caso, permite comprender la importancia de formarse hábitos ambientales saludables para resolver en gran medida el problema sobre el manejo, aprovechamiento y disposición inadecuados de los residuos sólidos inorgánicos generados en la comunidad. Por ejemplo, en lo que refiere al consumo de alimentos no procesados, estos deben tener valores mínimos, pues de lo contrario se estaría promoviendo la producción y comercialización de insumos industriales, pero sobre todo generando residuos sólidos difíciles de degradar, lo que termina aumentando la huella ecológica.

Estas aseveraciones guardan coincidencia con el planteamiento de Carpintero (2006), quien menciona la importancia de mantener la optimización en el uso de los recursos naturales (tierra y agua, recursos genéticos vegetales y animales, vegetación, suelos), para lograr una reducción de los impactos ambientales negativos. Sin embargo, este propósito representa un reto importante para los gobiernos, instituciones y comunidad en general, debido a que el modelo capitalista actual ha configurado diversas fuerzas de alto poder económico, que influyen sobre las formas de producción y consumo de alimentos porducidos a escala indusrtial.

Asi mismo, la utilización de la energía eléctrica en altas proporciones, demanda la utilización de otras materias no renovables como el gas natural, el carbón, entre otros elementos que cada vez son màs escasos, causando asi una alteración significativa de la geografía, pero sobre todo generando altas emisiones de $\mathrm{CO} 2$, que no sòlo contaminan el aire sino que afectan la capa de ozono y por ende alteran la estabilidad climática. Lo anterior guarda plena coincidencia con los planteamientos de Rivas (2012), quien manifiesta que los humanos vivimos en un planeta finito es decir que cuenta con recursos que son no renovables y la rapidez con la que están siendo explotados, representa una seria amenaza para la permanencia en la tierra.

Ahora bien, en lo que refiere la utilización del combustible, este es muy similar al anterior, dado que este recurso proviene de escavaciones de fósiles como el petróleo, además de 
alterar la geografía del planeta, contribuye a la contaminación de acuíferos subterráneos y como si estos fuera poco, la utilización en vehículos o maquinaria conduce a la liberación de C02 lo cual aumenta la huella ecológica. En total cincidencia Schneider y Samaniego (2009), manifiestan que la utilización de combustibles fósiles representan factor importante en el aumento de la huella ecológica, si se tiene encuenta que muchos de ellos se utilizan de manera recurrente en actividades como la calefacción, el transporte, la alimentación, entre otros.

De igual manera, en lo que refiere a generación de basuras, esta se asocia en gran medida al consumo de productos empacados o envasados, que provienen de la industria, no solo en el ámbito de la alimentación sino tambien para el aseo, el vestuario, el trabajo entre otras actividades cotidianas. Por consiguiente, los resultados obtenido en esta categoría coinciden con el hallazgo obtenido por Villalobos y Castillo (2015), quienes argumentan que la generación y sobre todo el manejo inadecuado de residuos sólidos es uno de los fenómenos que mas influye en el aumento de la huella ecológica, dado que muchos de sus empaques contienen materiales difíciles de degradar, además de que generan emisiones efecto invernadero que contribuyen en la alteración de los ecosistemas y el medio ambiente.

De manera general, los resultados obtenidos en el cálculo de la huella ecológica en el caso particular donde se obtuvo un valor de 4.7, representan un asunto preocupante, en la medida que se encuentra por encima de los promedios nacionales de 1.9, lo cual prende las alarmas de la manera como estamos haciendo uso de la capacidad instalada del planeta. Estos hallazgos también concuerdan con lo expresado por Contreras et al. (2019) en su estudio denominado "La Huella Ecológica, indicador de la responsabilidad social y ambiental de cara al 2030" desarrollado en Ecuador, donde se determinó que muchos de los participantes del estudio presentaron valores por encima del promedio nacional ubicado en el 2.8 .

Sin embargo, no todo esta perdido frente a esta realidad, dado que existen estudios que evidencian la posibilidad de reducir la huella ecológica personal, a partir de la implementación de estrategias en cuanto a los patrones de consumo para lograr un desarrollo sostenible. Por ejemplo Aliaga (2014) demuestra en uno de sus estudios cómo las estrategias aplicadas en un caso de estudios, permitieron disminuir significativamente la huella ecológica alcanzando unos valores de 1.13 ha a 0.898 ha. 
Por su parte, la organización no gubernamental World Wild Fund for Nature recomienda acciones relacionadas con los hábitos alimenticios y el consumo de los alimentos, pues un tercio de todos los alimentos, más de 1.430 millones de toneladas al año en todo el mundo, se desperdicia, lo que representa hasta el 10\% de las emisiones mundiales de gases de efecto invernadero, y aproximadamente una cuarta parte de toda la deforestación y el uso humano del agua (WWF, 2018).

Estos hallazgos motivan a encaminar a los individuos hacia un estilo de vida diferente, teniendo en cuenta que el hombre habita en un planeta que además de ser único presenta unas características muy particulares que debe protegerse para garantizar la existencia. De hecho la filosofia de reducción de la huella ecológica busca también un cambio de paradigma en el que educadores y estudiantes deben configurar una nueva manera de ver la tierra, pero sobre todo para comprender las relaciones existentes entre todos los elementos y recursos que la integran.

Ahora bien, a manera de cierre se pueden identificar un común denominador en los resultados encontrados en otros estudios en la medida que la mayoría coincide en que la educación cambie respecto a la huella ecológica implica un proceso de formación del individuo, para que este último pueda comprender las relaciones con el medio ambiente. De esta manera, se contribuye a la formación de conciencia social, para que los individuos reconozcan la importancia de protección de los recursos naturales y realizar un manejo adecuado de los desechos y residuos, con el fin de mantener un equilibrio sostenible, dado que continuamente surgen nuevos desafíos que requieren nuevas soluciones.

\section{CONCLUSIONES}

El enfoque de la huella ecológica como herramienta de gestión ambiental, permite situar la formación del individuo en un modelo mucho más flexible pero a su vez conservador, de cara a formar personas críticas, reflexivas y autónomas, capaces de hacer frente a las situaciones productivas que se gestan en el contexto, en su mayoría causante de los problemas ambientales que hoy persisten. Esta perspectiva resulta interesante, puesto que la escuela como institución promotora de bienestar, no puede ser ajena al cambio, cuando todo a su alrededor se encuentra en constante evolución, como consecuencia de los mismos avances tecnológicos, políticos y sociales de la comunidad.

Afrontar la reducción de la huella ecológica ambiental, implica un proceso de transformación de las actividades y la formación de hábitos para el buen manejo de los 
residuos y desechos, asi como en la optimización de los recursos naturales, de tal manera que sea posible llevar una vida sostenible en el planeta. Este enfoque se convierte en un enorme reto para las instituciones educativas, puesto que su práctica pedagógica ambiental es entendida como el espacio a la formación moral abordada en lo social, a fin de direccionar las acciones concretas de forma acertada acorde con las realidades culturales en el cual se desenvuelven los actores educativos, retomando las maneras de pensar y actuar, con el objetivo de construir los procesos de trasformación social encaminados al desarrollo sostenible.

La tarea de los educadores debe ir más allá de un ejecutor preparado para hacer efectiva cualquiera de las metas que se señale, puesto que es un ser intelectual con muchas libertades; capaz de crear espacios de aprendizaje donde se propicien los valores ambientales y el fortalecimiento de la conciencia frente al aprovechamiento de los recursos naturales de manera prudente, de tal manera que se garantice las condiciones de vida necesarias para las generaciones futuras.

Desde la perspectiva de la huella ecológica, la educación ambiental deja de ser mecanicista, y se establece un acto de autodeterminación del individuo, para que tome conciencia de sus formas de ver y estar en el mundo, además de tomar una postura crítica al modelo económico actual, mediante el rechazo de políticas que buscan la utilización intensiva de los recursos naturales, para lograr un desarrollo sostenible de la sociedad. De igual manera, la escuela está llamada a avanzar mediante la renovación de sus estructuras, para que pueda brindar espacios de aprendizaje donde se fortalezcan los hábitos ambientales y se alcance un cambio de paradigma y acabar con los vicios creados al interior de las comunidades como el consumismo, la deforestación, entre otros aspectos negativos que socaban el equilibrio ecológico.

\section{RECOMENDACIONES}

Para obtener beneficios verdaderos para empresas, organizaciones y para la comunidad en general, es necesario comenzar a aplicar estrategias que vayan disminuyendo los índices que más les generan la huella ecològica.

Todas las emisiones relacionadas a la compra de combustible, se puede pasar a un vehículo eléctrico, a la utilización de la bicicleta o compartir el vehículo con otros. 
En el consumo de energía, se pueden comprar electrodomésticos que sean de uso eficiente, usar iluminación led la cual consume menos energía, apoyar las campañas de sensibilización como apagar las luces y los computadores cuando no se estén utilizando. Hacr màs uso de la video llamada con el fin de evitar tantos desplazamientos en las salidas de negocio; y en cuanto a la generación de residuos es fundamental crear mucha conciencia entre los estudiantes y las personas de las organizaciones; disminuir el uso del papel en la vida cotidiana tanto en el hogar como en el trabajo, instituciones y oficinas. Para generar menos residuos, es importante hacer compras responsables de los alimentos y otros productos que hacen parte de la canasta familiar. Aplicar la estrategia de las tres $\mathrm{R}$ en el manejo, aprovechamiento y disposiciòn de los residuos sòlidos.

Con el fin de dar continuidad al proceso iniciado, se deben realizar las siguientes actividades: Se recomienda seguir utilizando el instrumento para la aplicación de la huella ecològica con una periodicidad de cada 2 meses durante un año, presentándose la facturación de la prestación de los servicios públicos de consumo de agua, energía eléctrica y en lo posible de gas domiciliario.

Es necesario realizar las respectivas comparaciones y comportamientos, además efectuar mediciones periódicas de los resultados obtenidos y su discusión.

\section{REFERENCIAS BIBLIOGRÁFICAS}

Aliaga, M. E. (2014). Determinación de la Huella Ecológica personal como estrategia para la adquisición de patrones de consumo sostenible UNCP. Saber y Hacer. Revista de Ingeniería de la USIL, 3(1), 49-69.

Bauman, Z. (2000) Liquid Modernity. Cambridge: Polity Press.

Balcazar, Fabricio E. (2003). Investigación acción participativa (iap): Aspectos conceptuales y dificultades de implementación.Fundamentos en Humanidades, vol. IV, núm. 7-8, pp. 59-77. Universidad Nacional de San Luis, Argentina. https://www.redalyc.org/pdf/184/18400804.pdf

Bohórquez Caldera, L. A. (2006). Huella ecológica y hábitos de consumo: el reto de la bioética frente al medio ambiente. Revista de la Universidad de La Salle, (42), 109-116.

https://ciencia.lasalle.edu.co/cgi/viewcontent.cgi?article=1299\&context=ruls

Carpintero, Ó. (2006). La huella ecológica de la agricultura y la alimentación en España, 1955-2000. Áreas. Revista Internacional de Ciencias Sociales, (25), 31-45. 
Contreras Velázquez, L. M., Guillén Pérez, L. y Formoso Mieres, A. (2019). Educaciòn, cultura y comunicación ambiental. La huella ecológica, indicador de la responsabilidad social y ambiental de cara al 2030. Educaciócultura. https://ambiente-sustentabilidad.org/index.php/revista/article/view/44

Delgado Cobas, L. (2013). La huella ecológica como herramienta en la gestión ambiental. https://go.gale.com/ps/i.do?id=GALE\%7CA448340003\&sid=googleScholar\&v= $\underline{2.1 \& \mathrm{it}=\mathrm{r} \& \text { linkaccess }=\mathrm{abs} \& \mathrm{issn}=00489115 \& \mathrm{p}=\mathrm{IFME} \& \mathrm{sw}=\mathrm{w} \& \text { userGroupName }}$ $=$ anon $\% 7 \mathrm{Ee} 8293 \mathrm{f0}$

Gàndara, A. S. (2011). Conceptos básicos de gestión ambiental y desarrollo sustentable. Instituto Nacional de Ecología.

Garrett, C. (2021). Huella ecológica: definición, cálculo y reducción https://climate.selectra.com/es/que-es/huella-ecologica\#la-huella-ecologica-porpaises-2021

Hernández Sampieri, R., Fernández Collado, C. y Baptista Lucio , P. (2006). Metodologia de la investigación. Sexta ed. Mc Graw Hill. http://observatorio.epacartagena.gov.co/ wp content/uploads/2017/08/metodologia-de-la-investigacion-sextaedicion.compressed.pdf

Jaramillo Henao, G., y Zapata Márquez, L. M. (2008). Aprovechamiento de los residuos sólidos orgánicos en Colombia. https://bibliotecadigital.udea.edu.co/ dspace/bitstream/10495/45/1/AprovechamientoRSOUenColombia.pdf

Martínez. (2004). ciencia y arte en la metodología cualitativa. Mexico: Trillas.

Martínez Castillo, R. (2007). Algunos aspectos de la huella ecológica. InterSedes: Revista de las Sedes Regionales, vol. VIII, núm. 14, 2007, pp. 11-25.Universidad de Costa Rica Ciudad Universitaria Carlos Monge Alfaro, Costa Rica. https://www.redalyc.org/pdf/666/66615071002.pdf

McAuliffe, M., y Khadria, B. (2019). Informe sobre las migraciones en el mundo 2020. Ginebra: Organización Internacional para las Migraciones. https://publications. iom. int/books/informe-sobre-las-migraciones-en-el-mundo-2020.

Palomares Ojeda, C. (2018). La huella ecológica cómo indicador de sostenibilidad ambiental. http://tauja.ujaen.es/bitstream/10953.1/8720/1/TFG.CeliaPalomaresOjeda.pdf 
Pérez Neira, D., De Marco Larrauri, O., y Álvarez Muñoz, P. (2015). La huella ecológica de las naciones. Reflexiones globales, particularidades ecuatorianas. Revista Ciencia UNEMI, 8(14), 93-103.

Programa de las Naciones Unidas para el Desarrollo.(s,f). Objetivos de Desarrollo Sostenible. https://www1.undp.org/content/undp/es/home/sustainabledevelopment-goals.html

Rivas, D. M. (2012). Energía y medio ambiente en un planeta finito. Mediterráneo económico, 22, 79-90.

Salazar Ortiz, V. H. (2018). Sobrepoblación y consumismo, principales retos para un desarrollo regional sustentable.

Sánchez, J., Domínguez, R., León, M., Samaniego, J., y Sunkel, O. (2019). Recursos naturales, medio ambiente y sostenibilidad: 70 años de pensamiento de la CEPAL.Schneider, H., y Samaniego, J. (2009). La huella del carbono en la producción, distribución y consumo de bienes y servicios. Santiago de Chile: Comisión Económica para América Latina y el Caribe (CEPAL), 29-34.

Torbisco Casals, N. (2000). Minorías culturales y derechos colectivos: un enfoque liberal. Universitat Pompeu Fabra.

Villalobos Perea, C. E. y Castillo Franco, C. A. ( 2015). Huella ecológica y gestión de residuos sólidos de la universidad autónoma de occidente presentado. https://campussostenible.org/wp-content/uploads/2017/04/anexo-11-huellaecologica-20,

Universidad Pontificia Bolivariana. (s,f). Sostenible ¿Cómo calcular la huella de carbono? (2019). https://www.upb.edu.co/es/central-blogs/sostenibilidad/como-calcularhuella-carbono

Universidad Pontificia Bolivariana. (2019). Estrategia UPB Sostenible.

World Wildlife Fund. (2014). Informe planeta vivo 2014. https://bit.ly/3ghdrYL World Wildlife Fund. (2018). Living Planet Report - 2018: Aiming Higher. https://bit.ly/2LSU7Dlpb.edu.co/es/central-blogs/sostenibilidad/como-calcularlar-huella-

Administrador/Downloads/WackernagelandGalli_2007.pdf:///F:/MODULO\%20 INVESTIGACION\%20III/DOCUMENTOS\%20ESNARE 